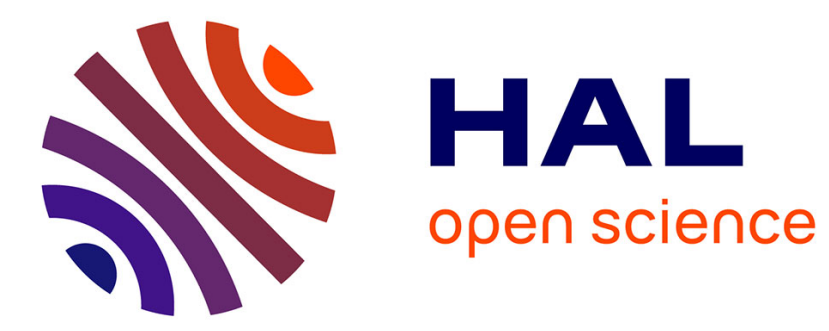

\title{
Portfolio Symmetry and Momentum
}

Monica Billio, Ludovic Calès, Dominique Guegan

\section{To cite this version:}

Monica Billio, Ludovic Calès, Dominique Guegan. Portfolio Symmetry and Momentum. 2009. halshs00363383v2

\section{HAL Id: halshs-00363383 \\ https://shs.hal.science/halshs-00363383v2}

Submitted on 30 Nov 2009

HAL is a multi-disciplinary open access archive for the deposit and dissemination of scientific research documents, whether they are published or not. The documents may come from teaching and research institutions in France or abroad, or from public or private research centers.
L'archive ouverte pluridisciplinaire HAL, est destinée au dépôt et à la diffusion de documents scientifiques de niveau recherche, publiés ou non, émanant des établissements d'enseignement et de recherche français ou étrangers, des laboratoires publics ou privés. 


\section{Documents de Travail du Centre d'Economie de la Sorbonne}

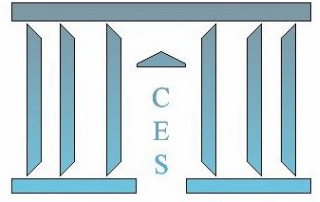

\section{Portfolio Symmetry and Momentum}

Monica BILlio, Ludovic CALÈS, Dominique GUEGAN

2009.03

Version révisée 


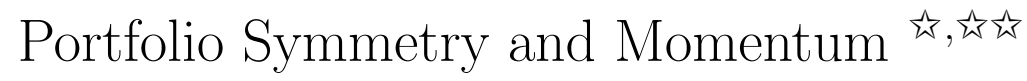

\author{
Monica BILLIO $^{\mathrm{a}}$, Ludovic CALÈS ${ }^{*, a, b}$, Dominique GUÉGAN ${ }^{\mathrm{b}}$ \\ ${ }^{a}$ University Ca' Foscari of Venice - Department of Economics \\ Fondamenta San Giobbe - Cannareggio 873 - 30121 Venice, Italy \\ ${ }^{b}$ Paris School of Economics - MSE-CES - University Paris-1 Panthéon-Sorbonne \\ 106, avenue de l'hopital - 75013 Paris, France
}

\begin{abstract}
This paper presents a novel theoretical framework to model the evolution of a dynamic portfolio (i.e., a portfolio whose weights vary over time), considering a given investment policy. The framework is based on graph theory and quantum probability. Embedding the dynamics of a portfolio into a graph, each node of the graph representing a plausible portfolio, we provide the probabilities for a dynamic portfolio to lie on different nodes of the graph, characterizing its optimality in terms of returns. The framework embeds cross-sectional phenomena, such as the momentum effect, in stochastic processes, using portfolios instead of individual stocks. We apply our methodology to an investment policy similar to the momentum strategy of Jeegadeesh and Titman (1993). We find that the strategy symmetry is a source of momentum.
\end{abstract}

Key words: (P) Finance, Graph Theory, Momentum, Quantum Probability, Spectral Analysis

JEL Classification: C14, C44

\section{Introduction}

In a seminal paper, Jeegadeesh and Titman (1993) introduce the momemtum strategy. Considering a given market, their strategy consists of

\footnotetext{
${ }^{4}$ An earlier version of this paper has been presented at a University Paris-1 seminar, in February 2009 and at the European Conference on Operational Research - EURO XXIII in July 2009. We thank participants for their comments and feedback.

whe authors are very grateful to Rodney Wolff and an anonymous referee for their numerous comments which greatly helped to improve the paper.

${ }^{*}$ Corresponding author.

Email addresses: billio@unive.it (Monica BILLIO), ludovic.cales@unive.it (Ludovic CALÈS), dguegan@univ-paris1.fr (Dominique GUÉGAN)
} 
buying stocks which performed well in the past and selling those which performed poorly. They showed that this strategy generates significant positive returns. This persistent arbitrage opportunity has raised a lot of interest, as it contradicts market efficiency.

The momentum strategy of Jeegadeesh and Titman (1993) is a zero-dollar long/short equally-weighted strategy being long the best stock decile of a given market and being short the worse stock decile. In order to determine the best and worse stock deciles, they rank the stocks according to their past performance. Typically, a stock past performance is its return over the last six months. The strategy being zero-dollar, the value of the sold stocks is the value of the bought stocks. The strategy being equally-weighted, the value invested in each stock is the same, in absolute value.

Jeegadeesh and Titman (1993) used their momentum strategy in a market composed of all available stocks from the $\mathrm{CRSP}^{1}$ database. This strategy has been used in many other markets. It has been shown to generate significant positive returns in most international stock markets (Rouwenhorst (1998), Chan et al. (2000)), in commodities markets (Kazemi et al. (2009)) and in currency markets (Okunev and White (2003)). For an extensive review of the research about momentum, we refer to Kazemi et al. (2009). Currently, it seems that momentum is a behavioral feature of finance. Indeed, according to Barberis et al. (1998) and Daniel et al. (1998), momentum is due to investors' under-reaction to news.

In this paper, we present a novel framework embedding cross-sectional phenomena, such as the momentum effect, in stochastic processes through the study of market dynamics using portfolios, and not individual stocks. Considering a given investment policy, we model the evolution of a dynamic portfolio as a path on a graph generated by an investment strategy. This framework takes into account the symmetries of the given strategy. These properties allows us to compute the probabilities for the occurences of given changes in a portfolio. Considering a momentum strategy, we find that the symmetry of this strategy is a source of the momentum effect. Finally, this new approach can improve asset management through the use of dynamic portfolios.

\footnotetext{
${ }^{1}$ Center for Reseach in Security Prices
} 
The paper is composed by four sections. Section 2 specifies the framework, based on graph theory and quantum probability, used to model the dynamics of a portfolio. In Section 3, we introduce the dynamics of a portfolio and its associated spectral distribution. Section 4 gives the probabilistic properties of the momentum effect and provides an application. Section 5 concludes.

\section{The Framework}

In this section, we introduce a framework which associates a graph with a given investment strategy.

\subsection{Jeegadeesg 85 Titman's Momentum Strategy}

An investment policy defines the set of all investable portfolios through the specification of constraints on the portfolios' weights. For instance, the momentum strategy of Jeegadeesh and Titman (1993) is defined as follows:

- $20 \%$ of stocks have non-zero weights (C1)

- the long and the short positions have equal weights, $\omega_{0}$, in absolute value $(\mathrm{C} 2)$

- the weights imply a 2:1 leverage $^{2}(\mathrm{C} 3)$

- the number of long and short positions is the same (C4)

We can specify these four properties in a more formal way. Let us consider a market of $N$ assets and note $\gamma(i)$ the given weight associated with asset $i$ in a portfolio. Respecting the momentum strategy of Jeegadeesh and Titman (1993), then we have:

- $\exists J \subset\{1, \ldots, N\}$ such that $\gamma(j)=0, \forall j \in J$ and $\frac{|J|}{N} \approx 0.8(\mathrm{C} 1)$

- $|\gamma(i)|=\omega_{0}, \forall i \in\{1, \ldots, N\} \backslash J, \omega_{0}>0 . \quad(\mathrm{C} 2)$

- $\sum_{i=1}^{N}|\gamma(i)|=2(\mathrm{C} 3)$

- $\sum_{i=1}^{N} \gamma(i)=0(\mathrm{C} 2+\mathrm{C} 4)$

\footnotetext{
${ }^{2}$ The notation 2:1 means that the amount of capital backing the portfolio represents $50 \%$ of the portfolio value. It is the minimum amount required under the U.S. Regulation $\mathrm{T}$. As a consequence, the sum of the absolute values of the weights of the portfolio equals 2 .
} 


\subsection{The Full Market Momentum Strategy}

Here we consider a momentum strategy slightly different from that of Jeegadeesh and Titman (1993) presented in Section 2.1, based on a zerodollar long/short strategy with a leverage of $2: 1$. Instead of having positions in two deciles of the market stocks, the strategy we consider has positions in each of the market stocks. The constraint (C1) in Section 2.1 becomes:

- $100 \%$ of the stocks have non-zero weights (C1)

So, any portfolio generated by this strategy is either long a position, with weight $\frac{2}{N}$, or short the same position, with weight $-\frac{2}{N}$. In the following, we denote by $\Gamma$ the set of portfolios generated by this specific strategy. We denote by $\gamma$ a portfolio of $\Gamma$ represented by the vector of its weights: $\gamma=(\gamma(1), \ldots, \gamma(N))^{\prime}$, where $A^{\prime}$ stands for the transpose of $A$, and $\gamma(i)$ corresponds to the weight of the portfolio $\gamma$ in the $i$ 'th asset, $\forall i=1, \ldots, N$.

We call this strategy the "full market momentum strategy with leverage 2:1".

Definition 1: Having a market of $N$ assets, $N$ even, we define $\Gamma$ to be the set of portfolios generated by the full-market momentum strategy with leverage $2: 1$, namely

$$
\Gamma=\left\{\gamma \in\left\{-\frac{2}{N}, \frac{2}{N}\right\}^{N} \mid \sum_{i=1}^{N} \gamma(i)=0\right\}
$$

\subsection{Strategy representation}

In order to obtain the distribution of the distance between a dynamic portfolio and its original portfolio - given a strategy and the portfolio dynamics - we use graph theory to obtain an elegant representation of the strategy. First of all, we specify the distance between two portfolios, then we introduce the notion of a graph associated with a strategy. Finally we illustrate our approach with an example.

Let $\gamma_{1}, \gamma_{2} \in \Gamma$. The turn-over between the two portfolios, noted $T O\left(\gamma_{1}, \gamma_{2}\right)$, defines a distance between $\gamma_{1}$ and $\gamma_{2}$ and we express it as the 1-norm distance:

$$
\operatorname{TO}\left(\gamma_{1}, \gamma_{2}\right)=\sum_{i=1}^{N}\left|\gamma_{1}(i)-\gamma_{2}(i)\right|
$$


In the case of the full market momentum strategy, this distance is important for the construction of the graph characterizing the strategy and for portfolio management. Indeed,

- the turn-over distance corresponds to the minimum number of permutations between long and short positions in order to pass from one portfolio to another. It is also known as the Damerau-Levenshtein distance, as in Damerau (1964) and Levenshtein (1966). Moreover, the previous distance describes the turn-over, and a distance of 1 can be interpreted as the minimum effort for an investor to pass from between portfolios. The minimization of the turn-over is of great interest for any investor because it reduces the transaction costs.

- it allows one to define a stratification of $\Gamma$, as presented in Hora and Obata (2007). Associated with three matrices (the quantum components), it leads to the quantum decomposition which is the main notion of quantum stochastic calculus. This aspect is beyond the scope of this paper but leads to the theorem 1 used in Section 3. For more details, we refer to Hora and Obata (2007), Chapter 2. A presentation of the stratification is provided in Section 2.4.

Now, we denote by $E$ the set of edges such that each edge binds two portfolios whose turnover is minimal, i.e.,

$$
E=\left\{\left\{\gamma_{1}, \gamma_{2}\right\} \mid T O\left(\gamma_{1}, \gamma_{2}\right)=1, \gamma_{1}, \gamma_{2} \in \Gamma\right\}
$$

Then, we denote by $G=(\Gamma, E)$ the undirected graph which characterizes the investment strategy. We provide an example for the full market momentum strategy, as follows. In a market of four assets (A, B, C and D), the portfolios generated by this strategy are given in Table 1 and the corresponding graph is given in Figure 1. The portfolios linked by an edge differ by one permutation between their long and short positions.

Minimization of the turn-over mentioned earlier corresponds to the search for the shortest path, which is a typical graph theory problem. 


\begin{tabular}{|r|r|r|r|r|}
\hline & Asset A & Asset B & Asset C & Asset D \\
\hline$\gamma_{1}$ & $2 / N$ & $2 / N$ & $-2 / N$ & $-2 / N$ \\
\hline$\gamma_{2}$ & $-2 / N$ & $2 / N$ & $-2 / N$ & $2 / N$ \\
\hline$\gamma_{3}$ & $2 / N$ & $-2 / N$ & $-2 / N$ & $2 / N$ \\
\hline$\gamma_{4}$ & $-2 / N$ & $2 / N$ & $2 / N$ & $-2 / N$ \\
\hline$\gamma_{5}$ & $2 / N$ & $-2 / N$ & $-2 / N$ & $2 / N$ \\
\hline$\gamma_{6}$ & $-2 / N$ & $-2 / N$ & $2 / N$ & $2 / N$ \\
\hline
\end{tabular}

Table 1: Portfolios of the Full Market Momentum strategy in a market of four assets

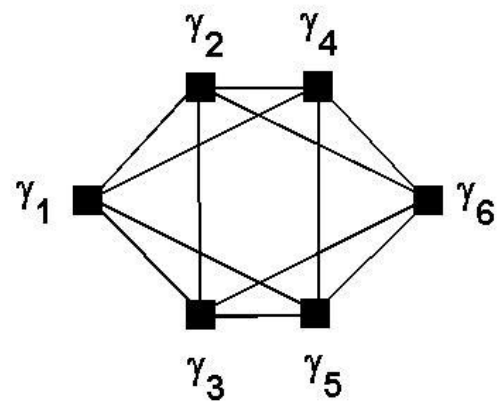

Figure 1: Graph associated with the Full Market Momentum Strategy

\subsection{The stratification of $\Gamma$}

We now present the stratification of $\Gamma$ discussed previously. Let us consider a given portfolio $\gamma^{*} \in \Gamma$. Then, the disjoint union of strata $\Gamma_{n}$ such that

$$
\Gamma=\bigcup_{n=0}^{\infty} \Gamma_{n}, \Gamma_{n}=\left\{\gamma \in \Gamma \mid T O\left(\gamma, \gamma^{*}\right)=n\right\}
$$

is the stratification of $\Gamma$ associated with the portfolio $\gamma^{*}$, given by the minimum number of permutations which separate $\gamma^{*}$ from the portfolios of each stratum.

Considering the previous example, the stratification of $\Gamma$ associated with $\gamma_{1}$ is illustrated in Figure 2, with

$$
\Gamma_{0}=\left\{\gamma_{1}\right\}, \Gamma_{1}=\left\{\gamma_{2}, \gamma_{3}, \gamma_{4}, \gamma_{5}\right\}, \Gamma_{2}=\left\{\gamma_{6}\right\}
$$




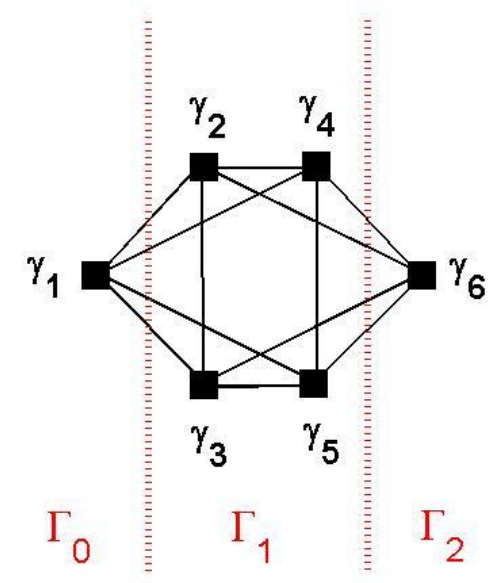

Figure 2: Stratification of $\Gamma$ associated with portfolio $\gamma_{1}$

Now, we introduce the optimal portfolio of $\Gamma$ over a given period. Given the returns of $N$ assets over a given period, we define the optimal portfolio for these assets as the portfolio, among those in $\Gamma$, which provides the highest return. In the case of the full market momentum strategy, the optimal portfolio buys the $\frac{N}{2}$ assets which performed the best and sells the $\frac{N}{2}$ assets which performed the worst.

An important stratification for the study of the momentum effect is the one associated with the optimal portfolio in the case of the full market momentum strategy. The returns of the portfolios generated by this strategy, relative to the return of the optimal portfolio and according to the portfolios' strata, have the distribution given in Figure 3. The return of the portfolios is defined relative to the optimal portfolio return, so it is normalized over the different periods.

In Figure 3, we consider the monthly returns of the ten world sectorial indices of Datastream from 01/1973 to 09/2009 (439 observations per index). In the abscissa, we indicate the ratio of the portfolios' returns with respect to the optimal portfolio return. We observe that, on average, the return of a portfolio of a stratum $n$ is greater than the one of a portfolio of a stratum $m$ when $m>n$. Consequently, we expect a portfolio which is at a distance 
$n$ from the optimal portfolio to provide a return greater than the return of a portfolio which is at a distance $m$ from the optimal portfolio, when $m>n$. This fact is the basis of the study of the momentum effect.

We also observe that the distributions of the returns of the portfolios corresponding to the strata $\Gamma_{n}$ and $\Gamma_{\frac{N}{2}-n}$ are symmetric with respect to 0 . This property is due to the symmetry of $\Gamma$ : indeed, if $\gamma \in \Gamma_{n}$, then $-\gamma \in \Gamma_{\frac{N}{2}-n}$.

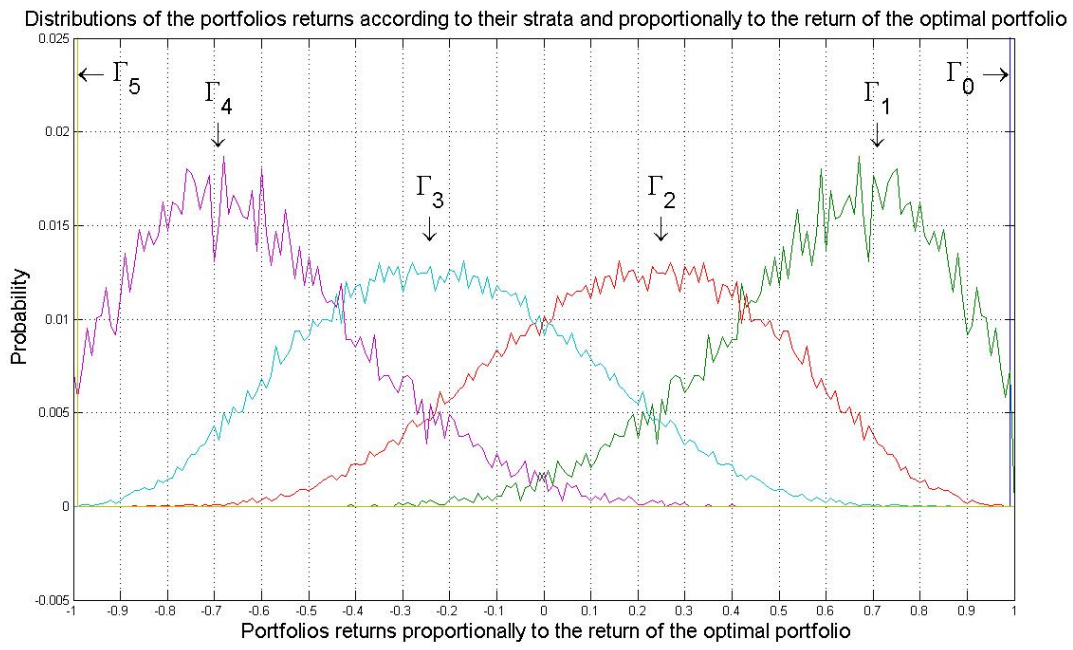

Figure 3: Distribution of the portfolios returns according to the portfolios' strata and relatively to the return of the optimal portfolio

\subsection{Dynamic Portfolio}

A dynamic portfolio is a "portfolio" whose weights change over time. We represent it as a sequence of portfolios. For instance, in a market of four assets, a dynamic portfolio over the periods $\left\{T_{1}, \ldots, T_{5}\right\}$ can be represnted by $\left(\gamma_{T_{1}}, \gamma_{T_{2}}, \gamma_{T_{3}}, \gamma_{T_{4}}, \gamma_{T_{5}}\right)$, where

$$
\begin{gathered}
\gamma_{T_{1}}=\left(\begin{array}{r}
2 / \mathrm{N} \\
2 / \mathrm{N} \\
-2 / \mathrm{N} \\
-2 / \mathrm{N}
\end{array}\right) ; \gamma_{T_{2}}=\left(\begin{array}{r}
2 / \mathrm{N} \\
-2 / \mathrm{N} \\
-2 / \mathrm{N} \\
2 / \mathrm{N}
\end{array}\right) ; \gamma_{T_{3}}=\left(\begin{array}{r}
2 / \mathrm{N} \\
-2 / \mathrm{N} \\
2 / \mathrm{N} \\
-2 / \mathrm{N}
\end{array}\right) . \\
\gamma_{T_{4}}=\left(\begin{array}{r}
-2 / \mathrm{N} \\
-2 / \mathrm{N} \\
2 / \mathrm{N} \\
2 / \mathrm{N}
\end{array}\right) ; \gamma_{T_{5}}=\left(\begin{array}{r}
-2 / \mathrm{N} \\
2 / \mathrm{N} \\
2 / \mathrm{N} \\
-2 / \mathrm{N}
\end{array}\right)
\end{gathered}
$$


We call $\gamma_{T_{1}}$ the origin portfolio for the dynamic portfolio $\left(\gamma_{T_{1}}, \gamma_{T_{2}}, \gamma_{T_{3}}, \gamma_{T_{4}}, \gamma_{T_{5}}\right)$. The periods $\left\{T_{1}, \ldots, T_{5}\right\}$ can overlap, such as being periods corresponding to a rolling window. An example of dynamic portfolio is the sequence of optimal portfolios over a rolling window. Another one is the sequence of portfolios of $\Gamma$ with the lowest volatility over a rolling window.

\section{The Spectral Distribution}

Given a graph associated with a strategy, we consider a dynamic portoflio whose dynamics is characterized by a random walk and we compute the distribution associated with the distance between this portfolio and its origin. This distribution is called the "spectral distribution".

\subsection{Portfolio dynamics: a random walk}

First, we suppose that the dynamic portfolio follows a walk on the graph $G=(\Gamma, E)$. Thus, we have the following assumption:

$\mathbf{A}_{0}$ : Considering a rolling window $\left(T_{1}, \ldots, T_{n}\right)$ where each $T_{i}$ has the same length $T$, then we say that the dynamic portfolio $\left(\gamma_{T_{1}}, \ldots, \gamma_{T_{n}}\right)$ follows a walk on the graph $G=(\Gamma, E)$ if $T O\left(\gamma_{T_{i}}, \gamma_{T_{i+1}}\right) \leq 1, \forall i \in\{1, \ldots, n\}$.

In the case of the full market momentum strategy, this means that two consecutive portfolios differ by at most one permutation. Figure 4 illustrates such a walk. This hypothesis is relevant for a dynamic portfolio based on returns whenever we expect that two consecutive portfolios with overlapping periods are very close. By choosing a long period and a short frequency, it appears possible to make this difference very small.

As an example, let us consider the market constituted by the ten Datastream world indices ${ }^{3}$ over 439 months from 01/1973 to 09/2009. In this market, we consider the optimal dynamic portfolio of the full market momentum strategy over a 22-day rolling window (period: 22 days; frequency: daily), and

we count the differences between two consecutive optimal portfolios. The results are given in Table 2 .

\footnotetext{
${ }^{3}$ WORLD-DS Oil \& Gas, Basic Mats, Industrials, Consumer Gds, Health Care, Consumer Svs, Telecom, Utilities, Financials, Technology
} 


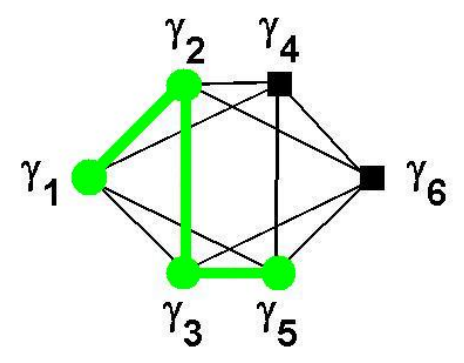

Figure 4: Illustration of a walk in a graph

\begin{tabular}{|l|c|c|c|c|}
\hline Distance & 0 & 1 & 2 & $3+$ \\
\hline Percentage of occurence & $56 \%$ & $41 \%$ & $3 \%$ & $0 \%$ \\
\hline
\end{tabular}

Table 2: Distance between two consecutive portfolios in the optimal dynamic portfolio

We observe that the assumption $\mathbf{A}_{\mathbf{0}}$ is violated $3 \%$ of the time, and only by a distance of 2 . We can fairly suppose that, in the case of a rolling window with an higher frequency (for instance hourly data), an intermediate portfolio appears. Then, instead of having two portfolios $\left(\gamma_{a}, \gamma_{b}\right)$ such that $\operatorname{TO}\left(\gamma_{a}, \gamma_{b}\right)=2$, we shall have three portfolios $\left(\gamma_{a}, \gamma_{c}, \gamma_{b}\right)$ such that $T O\left(\gamma_{a}, \gamma_{c}\right)=T O\left(\gamma_{c}, \gamma_{b}\right)=1$. Thus, the $3 \%$ of violations will diminish. Moreover, we are only interested in the moves of a dynamic portfolio, thus, when two successive portfolios are equal we drop the second one.

We suppose now that the dynamics of the portfolio is characterized by a random walk with an equal probability of moving in any direction, thus we introduce the following assumption:

$\mathbf{A}_{\mathbf{1}}$ : The dynamic portfolio $\gamma$ follows a random walk with an equal probability of moving in any direction.

In the case of the full market momentum strategy, this means that we give the same probability for the occurence of any permutation. 


\subsection{The Spectral Distribution of a Dynamic Portfolio}

We give now an expression for the spectral distribution associated with this dynamic portfolio. The spectral distribution presented below corresponds to the probability of finding a dynamic portfolio at a given distance from an origin portfolio: it provides the probabilities for the dynamic portfolio to be in different strata of $\Gamma$.

\subsubsection{General expression for the spectral distribution}

Consider a graph $G=(\Gamma, E)$ generated by a given strategy. We know that if the graph $\mathrm{G}$ is 2-antipodal distance-regular ${ }^{4}$, then it can be characterized by the eigenvalues of its adjacency matrix, and their multiplicities, Fiol et al. (1996) . It means that the properties of an investment strategy represented by such a graph can also be resumed by its eigevalues. Consider now a dynamic portfolio $\gamma_{p}$ which verifies the hypothesis $\mathbf{A}_{\mathbf{1}}$, on a distance-regular graph, then the following theorem provides the expression of the spectral distribution characterizing this dynamic portfolio. The proof of this theorem is postponed in Section 6.3.

Theorem 1. If $G$ is a finite distance-regular graph with diameter $d$ and intersection table

$$
\left(\begin{array}{ccccc}
c_{0} & c_{1} & c_{2} & \ldots & c_{d} \\
a_{0} & a_{1} & a_{2} & \ldots & a_{d} \\
b_{0} & b_{1} & b_{2} & \ldots & b_{d}
\end{array}\right)
$$

then the spectral distribution of $G$ is a probability measure such that the probability for dynamic portfolio $\gamma_{p}$ verifying $\mathbf{A}_{\mathbf{1}}$ to be at a distance $n$ from the original portfolio $\gamma_{o}$ after $p$ random moves on $G$ is :

$$
P\left(T O\left(\gamma_{p}, \gamma_{o}\right)=n\right)=\frac{\sqrt{\left|\Gamma_{k}\right|}}{d^{2 p} \| P_{k}|||\Gamma|} \sum_{i=0}^{d} m_{i} \lambda_{i}^{p} P_{k}\left(\lambda_{i}\right)
$$

where $\lambda_{i}$ are the eigenvalues of the adjacency matrix of the graph $G$, with their multiplicities $m_{i}$, and $\left\{P_{n}(x)\right\}_{n=0}^{d}$ are the Gram-Schmidt polynomials, defined as below in the three-term recurrence relation:

\footnotetext{
${ }^{4}$ These notions are recalled in Section 6
} 


$$
\begin{aligned}
& P_{0}(x)=1 \\
& P_{1}(x)=x-a_{0} \\
& x P_{n}(x)=P_{n+1}(x)+a_{n} P_{n}(x)+b_{n-1} c_{n} P_{n-1}(x), n=1,2, \ldots
\end{aligned}
$$

This theorem is the discrete classical version of a continuous-time theorem presented in Salimi (2009). The first use of the distance-regular property to characterize a walk is provided in Jafarizadeh and Salimi (2007) for a continuous-time quantum walk. This continuous-time quantum version can also be found as theorem 14.2.1 (and corollary 14.2.2) in the lecture given by Obata in Wroclaw in $2008^{5}$.

\subsubsection{The spectral distribution of a dynamic portfolio in the case of a full- market momentum strategy}

We compute now the spectral distribution provided by the previous theorem for a full-market momentum strategy. Consider $G=(\Gamma, E)$ the graph generated by the full-market momentum strategy on a market of $N$ assets. In order to apply Theorem 1 , we need to verify that $G$ is a distance-regular graph and we also need to compute the eigenvalues of its adjacency matrix, with their multiplicities. This last computation uses the 2-antipodal property of $G$. These properties are obtained in the following lemma whose proof is relegated to Section 6, with the computations of the eigenvalues of the adjacent matrix of $G$.

Lemma 1. Given a graph generated by the full market momentum strategy, then we have the following properties:

(i) It is a distance-regular graph,

(ii) It is a 2-antipodal graph.

Thanks to this lemma, we are able to compute the spectral distribution associated with a full market momentum strategy. We illustrate this result in the following example. We consider a market of ten assets $(N=10)$ and we assume that a dynamic portfolio follows a random walk on $G=(\Gamma, E)$. We provide in Figure 5 the probabilities that it will be at distance $n$ from its origin after $p=1,3,5,10$ moves. They correspond to the probabilities of the dynamic portfolio to be in the stratum $\Gamma_{n}$ after $p=1,3,5,10$ moves.

\footnotetext{
${ }^{5}$ The notes of this lecture are available at http://www.math.uni.wroc.pl/analiza/obata/index.html
} 


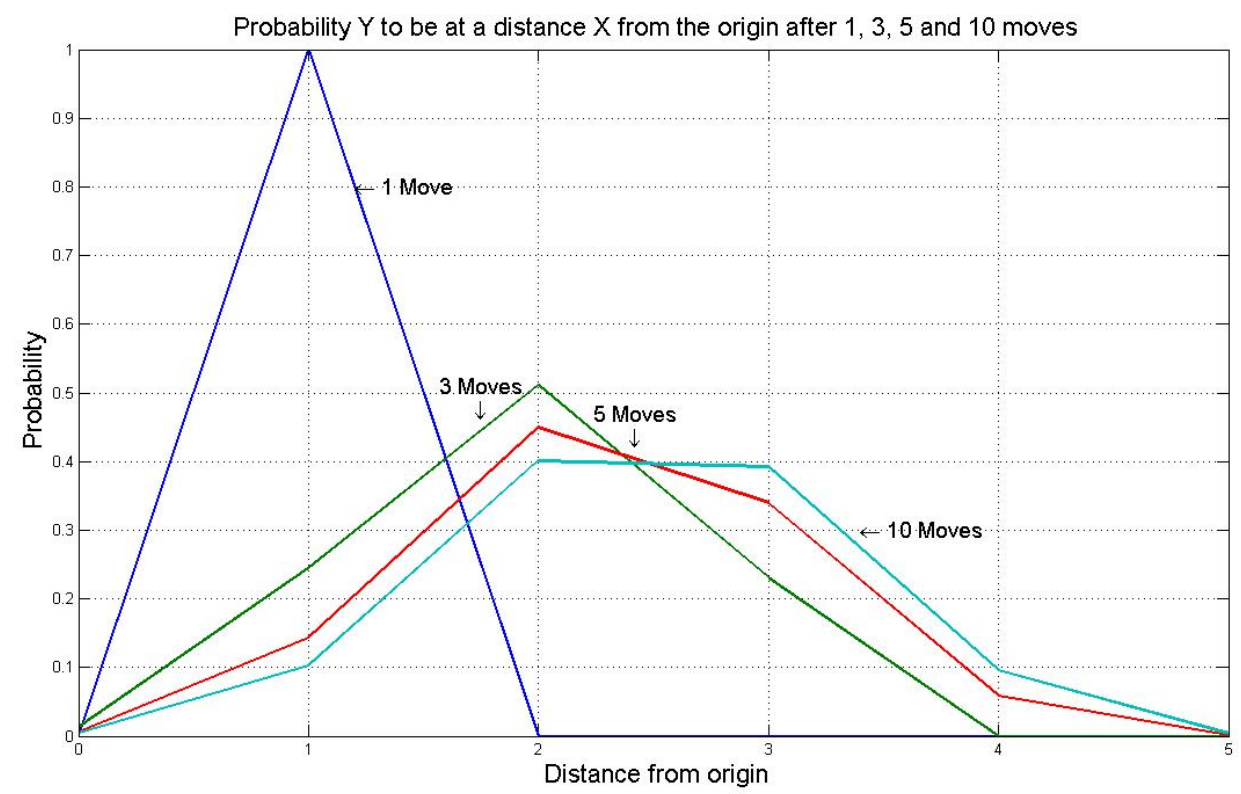

Figure 5: Spectral distribution for a dynamic portfolio to be at a distance $n$ from its origin after $1,3,5,10$ moves

After one move, the dynamic portfolio can only be at a distance 1 from its origin, i.e., in stratum $\Gamma_{1}$. After three moves, the dynamic portfolio can only be observed at distance $0,1,2$ or 3 from its origin. Its distribution is then clearly skewed toward 0 . The larger the number of moves, the more the spectral distribution tends to be symmetric, and thus less skewed toward zero. After ten moves, the distribution is already quite symmetric. Intuitively, when the number of moves increases, the probability of finding the dynamic portfolio in a node tends to be the same for any node. So, $P\left(T O\left(\gamma_{p}, \gamma_{o}\right)=n\right)$ tends to $\frac{\left|\Gamma_{n}\right|}{|\Gamma|}$ when $p$ tends to infinity.

\section{The Full Market Momentum strategy and the Momentum effect}

In this section, we propose linking the momentum effect with the evolution of the optimal dynamic portfolio. Then, we illustrate this result through the market of the ten Datastream world indices discussed above. 


\subsection{The Momentum Effect}

The momentum effect characterizes the persistance of the returns of a given portfolio. Jeegadeesh and Titman (1993) showed empirically that the optimal dynamic portfolio of their investment strategy (outlined in Section 2.1) presents some momentum effect. With our approach, considering that the assets selected in their strategy constitute the market, we observe the same momentum effect as them. Thus, as soon as we are in presence of the momentum effect, the optimal portfolio of our strategy should provide a positive return over the next period.

In Section 2.4, we have seen that we can characterize the momentum effect in terms of distance instead of returns. Thus, the new optimal portfolio should be close rather than far from the previous optimal portfolio. In other words, the spectral distribution of the optimal dynamic portfolio should be skewed toward 0. So, knowing the distance of a given portfolio from the origin portfolio is important because it permits one to measure how far the previous optimal portfolio (used as the investment portfolio) is from the current optimal portfolio. The shorter this distance, the greater are the returns of the investment portfolio, and the greater is the momentum effect.

\subsection{Empirical verification with a ten-assets market}

Consider the market constituted by the ten Datastream world indices over 439 months from 01/1973 to 09/2009. In this market, applying the full market momentum strategy, i.e., buying the half best performers and selling the half worse performers of the last month, and holding them the next month, leads to a monthly price return of $0.39 \%$ with a $t$-statistic of 3.47 . So, this market exhibits momentum.

To describe the evolution of the optimal portfolio of the full-market momentum strategy, month by month, we use the spectral distribution given in Theorem 1. Indeed, we assume that the dynamic portfolio satisfies assumption $\mathbf{A}_{\mathbf{1}}$. The probabilities for the dynamic portfolio to be at a distance $n$ from its origin after $p=1,3,5,10$ moves are illustrated in Figure 5 .

In Figure 6, we report the number of single steps that this optimal dynamic portfolio has executed every month. We observe that the number of moves is not always large enough to lead to a symmetric spectral distribution. Indeed, $8.9 \%$ of the number of moves are less than or equal to 5 , and $17.8 \%$ of the 
moves are less than or equal to 6 . They correspond to probabilities $60 \%$ and $56 \%$ of being at a distance less than or equal to 2 from the origin portfolio. So, the spectral distribution is clearly skewed. On average, it leads to a positive return for the dynamic portfolio, as we have shown in Section 2.4. So, according to our modeling, we observe momentum for about $15 \%$ of the observations. For the rest of the time, the number of moves leads to a symmetric distribution which corresponds to equal probabilities for the final portfolio to provide positive or negative returns. As a consequence and overall, we consider the strategy to be profitable.

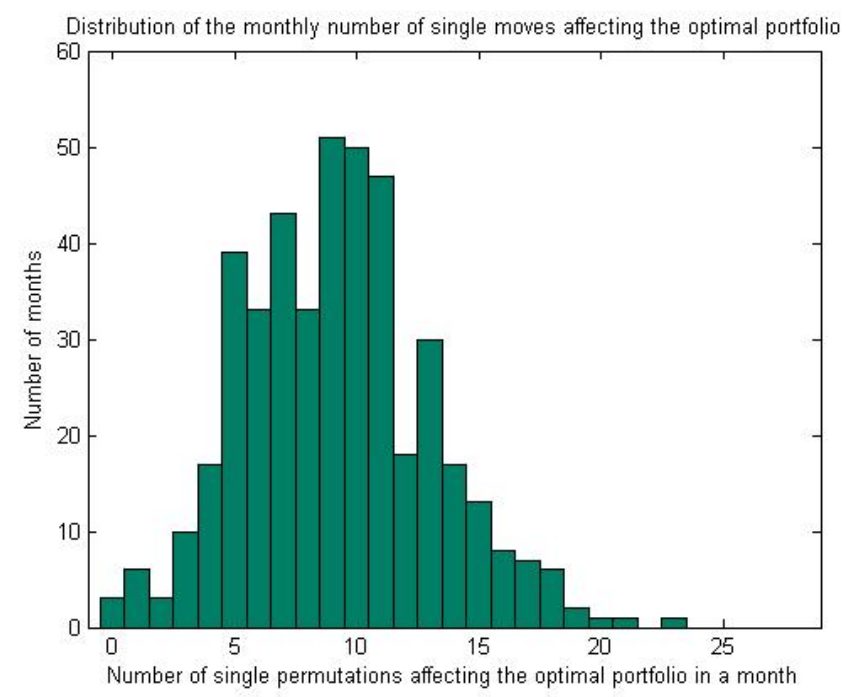

Figure 6: Number of single moves of the optimal portfolios over a month

In Section 3.2.2, we have seen that the symmetry of the full market momentum strategy, through its graph, implies that the spectral distribution of a dynamic portfolio following a random walk is skewed towards its origin and that only for large numbers of moves it tends to be symmetric. Moreover, from our previous illustration, the number of moves observed in the market is not always large enough to imply a symmetric spectral distribution. So, in this case, the symmetry of the full market momentum strategy implies at least a part of the momentum effect observed on the market. 


\section{Conclusion}

In this paper, we propose a novel framework to model the walk of dynamic portfolios on graphs corresponding to determined strategies. The nodes consist of the investable portfolios generated by a given strategy and the edges bind the portfolios which are at the minimal turn-over distance from one to the other. Under the assumption that a dynamic portfolio evolves passing from a portfolio of the graph to an adjacent one, we can use recent developments in graph theory and quantum probability to get the spectral distribution of the dynamic portfolio to be at a certain distance from a previous location. These probabilities depend on the graph's eigenvalues which represents the symmetries of the graph, i.e., the symmetries of the investment strategy.

As an illustration, we have seen that the symmetry of the momentum strategy induces a part of the momentum effect. Indeed a random walk on the graph generated by such a strategy produces probabilities (for the dynamic portfolio to be at a certain distance from a previous location) skewed enough to be at the origin of the momentum effect. Moreover, part of the momentum effect observed in the momentum strategy of Jeegadeesh and Titman (1993) can also be explained by this property of symmetry which characterizes the momentum strategy. Indeed, considering as the entire market the one composed of the assets selected in the strategy of Jeegadeesh and Titman (1993), the conclusions obtained for the full-market momentum strategy stand.

As the momentum effect is a short-term property of the markets, it would also be interesting, as future work, to model trends as long run walks, considering random walks as the noise surrounding this long run walk. Eventually, one might even think about modeling a business cycle as a cycle in terms of walk on a graph.

\section{References}

Barberis N, Shleifer A, Vishny R. A model of investor sentiment. Journal of Financial Economics 1998; 49; 307-343

Chan K, Alaudeen H, Wilson T. Profitability of momentum strategies in the international equity markets. Journal of Financial and Quantitative Analysis 2000; 35; 153-172 
Dalfó C, Fiol M, Garriga E. On k-walk-regular graphs. The Electronic Journal of Combinatorics 2009; 16; 1-9

Damerau, F.J. A technique for computer detection and correction of spelling errors. Communications of the ACM 1964

Daniel K, Hirshleifer D, Subrahmanyam A. Investor Psychology and security under- and over-reactions. Journal of Finance 1998; 53; 1839-1885

Fiol M, Garriga E, Yebra J. Locally pseudo-distance-regular graphs. Journal of Combinatory Theory B 1996; 68; 178-205

Hora A, Obata N. Quantum Probability and Spectral Analysis of Graphs. Springer-Verlag; 2007.

Jafarizadeha M. A., Salimia S. Investigation of Continuous-Time Quantum Walk Via Spectral Distribution associated with Adjacency Matrix. Annals of Physics 2007, ; 322-5; 1005-1033

Jeegadeesh N, Titman S. Returns to buying winners and selling losers: Implications for stock market efficiency. Journal of Finance 1993; 48; 65-91

Kazemi H, Schneeweis T, Spurgin R. Momentum in asset returns: are commodity returns a special case? Journal of Alternative Investments 10 (2009) 23-36

Levenshtein, V. I. Binary codes capable of correcting deletions, insertions, and reversals. Soviet Physics Doklady 1966; 10; 707-710

Obata N. Quantum Probability and Applications to Complex Networks Lecture notes - Wroclaw University

Okunev K, White D. Do momentum-based strategies still work in foreign currency markets ? Journal of Financial and Quantitative Analysis 2003; $38 ; 425-447$

Rouwenhorst K. International Momentum Strategies. Journal of Finance $1998 ; 53 ; 267-284$

Salimi S. Continuous-time quantum walks on semi-regular spidernet graphs via quantum probability theory. Working Paper; 2009; 


\section{APPENDIX}

\subsection{Proof of Lemma 1}

(i) Let denote $\mathrm{G}$ the graph generated by the Full Market Momentum Strategy in a market of $\mathrm{N}$ assets. We suppose $\mathrm{N}$ even. Let $i, j, k$ be non-negative integers. We know that a graph $G=(V, E)$ is distance-regular if for any choice of $x, y \in V$ with $d(x, y)=k$ the number of vertices $z \in V$ such that $d(x, z)=i$ and $d(y, z)=j$ is independent of the choice of $x, y$. Thus, taking $x, y \in V$ with $d(x, y)=k$ the number $p_{i j}^{k}=|\{z \in V ; d(x, z)=i, d(y, z)=j\}|$ is defined only depending on $i, j, k$.

Let $F_{2}^{N}$ be the binary vector space of dimension $\mathrm{N}$. As we are interested in a two-weight strategy, there exists an isomorphism between $\Gamma$ and a subset of $F_{2}^{N}$. We consider that a vector $\phi$ of $F_{2}^{N}$ represents a portfolio $\gamma$ with $\gamma(i)=-\frac{2}{N}$ if $\phi(i)=0$ and $\gamma(i)=\frac{2}{N}$ if $\phi(i)=1, i \in\{1, \ldots, N\}$.

Let consider $\gamma, \gamma_{1}, \gamma_{2} \in \Gamma$, three portfolios such that $d\left(\gamma, \gamma_{1}\right)=i, d\left(\gamma, \gamma_{2}\right)=j$ and $d\left(\gamma_{1}, \gamma_{2}\right)=k$. We note $\phi, \phi_{1}$ and $\phi_{2}$ the respective representions of $\gamma$, $\gamma_{1}$ and $\gamma_{2}$ in $F_{2}^{N}$. Let note $\Gamma_{i}\left(\gamma_{1}\right)$ and $\Gamma_{j}\left(\gamma_{2}\right)$ the sets of the portfolios which are, respectively, at a distance $\mathrm{i}$ from $\gamma_{1}$ and at a distance $\mathrm{j}$ from $\gamma_{2}$.

$$
\begin{gathered}
\Gamma_{i}\left(\gamma_{1}\right)=\left\{\gamma \in \Gamma \mid d\left(\gamma_{1}, \gamma\right)=i\right\} \\
\Gamma_{j}\left(\gamma_{2}\right)=\left\{\gamma \in \Gamma \mid d\left(\gamma_{2}, \gamma\right)=j\right\}
\end{gathered}
$$

We divide $\Gamma_{i}\left(\gamma_{1}\right) \cap \Gamma_{j}\left(\gamma_{2}\right)$ in 4 parts:

$$
\begin{aligned}
& P_{1}=\left\{i \in\{1, \ldots, N\} \mid \phi_{1}(i)=0 \text { and } \phi_{2}(i)=1\right\} \\
& P_{2}=\left\{i \in\{1, \ldots, N\} \mid \phi_{1}(i)=1 \text { and } \phi_{2}(i)=0\right\} \\
& P_{3}=\left\{i \in\{1, \ldots, N\} \mid \phi_{1}(i)=0 \text { and } \phi_{2}(i)=0\right\} \\
& P_{4}=\left\{i \in\{1, \ldots, N\} \mid \phi_{1}(i)=1 \text { and } \phi_{2}(i)=1\right\} .
\end{aligned}
$$

As $d\left(\gamma_{1}, \gamma_{2}\right)=k$, we have:

$$
\left|P_{1}\right|=k,\left|P_{2}\right|=k,\left|P_{3}\right|=\frac{N}{2}-k \text { and }\left|P_{4}\right|=\frac{N}{2}-k .
$$

Now, we study the allocation of, let say, the long positions of $\gamma$ among these 4 parts. The strategy is two-weighted so, once the long positions are allocated, the short positions also are.

Let note: 


$$
\begin{aligned}
& A=\left\{i \in\{1, \ldots, N\} \mid \phi(i)=1 \text { and } \phi_{1}(i)=0 \text { and } \phi_{2}(i)=1\right\} \\
& B=\left\{i \in\{1, \ldots, N\} \mid \phi(i)=1 \text { and } \phi_{1}(i)=1 \text { and } \phi_{2}(i)=0\right\} \\
& C=\left\{i \in\{1, \ldots, N\} \mid \phi(i)=1 \text { and } \phi_{1}(i)=0 \text { and } \phi_{2}(i)=0\right\} \\
& D=\left\{i \in\{1, \ldots, N\} \mid \phi(i)=1 \text { and } \phi_{1}(i)=1 \text { and } \phi_{2}(i)=1\right\}
\end{aligned}
$$

With these notations, we have:

$$
\left|\Gamma_{i}\left(\gamma_{1}\right) \cap \Gamma_{j}\left(\gamma_{2}\right)\right|=\sum_{|A|,|B|,|C|,|D|}\left(\begin{array}{c}
k \\
|A|
\end{array}\right)\left(\begin{array}{c}
k \\
|B|
\end{array}\right)\left(\begin{array}{c}
\frac{N}{2}-k \\
|C|
\end{array}\right)\left(\begin{array}{c}
\frac{N}{2}-k \\
|D|
\end{array}\right)
$$

The strategy is a full-market momentum strategy, so $\phi$ has $\frac{N}{2}$ long positions and $\frac{N}{2}$ short positions, so:

$$
|A|+|B|+|C|+|D|=\frac{N}{2} .
$$

Moreover $d\left(\gamma, \gamma_{1}\right)=i$, so we have:

$$
|A|+|C|=i .
$$

As $d\left(\gamma, \gamma_{2}\right)=j$, we also have:

$$
|B|+|C|=j
$$

From (3) and (4), we get:

$$
\begin{gathered}
|B|=|A|+j-i \\
|C|=i-|A| .
\end{gathered}
$$

From (2), (5) and (6), we get:

$$
|D|=\frac{N}{2}-j-|A| .
$$

Noting $|A|$ and inserting (5), (6) and (7) in (1), we finally get:

$$
\begin{aligned}
\left|\Gamma_{i}\left(\gamma_{1}\right) \cap \Gamma_{j}\left(\gamma_{2}\right)\right| & =\sum_{n}\left(\begin{array}{c}
k \\
n
\end{array}\right)\left(\begin{array}{c}
k \\
n+j-i
\end{array}\right)\left(\begin{array}{c}
\frac{N}{2}-k \\
i-n
\end{array}\right)\left(\begin{array}{c}
\frac{N}{2}-k \\
\frac{N}{2}-j-n
\end{array}\right) \\
& =\sum_{n=0}^{k-j+i}\left(\begin{array}{c}
k \\
n
\end{array}\right)\left(\begin{array}{c}
k \\
k+i-j-n
\end{array}\right)\left(\begin{array}{c}
\frac{N}{2}-k \\
i-n
\end{array}\right)\left(\begin{array}{c}
\frac{N}{2}-k \\
n+j-k
\end{array}\right)
\end{aligned}
$$


From (8), we have that $\left|\Gamma_{i}\left(\gamma_{1}\right) \cap \Gamma_{j}\left(\gamma_{2}\right)\right|$ only depends on $\mathrm{i}, \mathrm{j}$ and $\mathrm{k}$, thus $\mathrm{G}$ is a distance-regular graph.

(ii) A graph $\mathrm{G}$ is k-antipodal when each of its vertex is diametrically opposed to k-1 others which are themselves diametrically opposed.

Considering the graph defined by a Full-Market Momentum strategy, the distance considered (half the Hamming distance) consists in permutations between long and short positions. Then, the maximum number of permutations that can be applied to a given portfolio is obtained by permuting all its long position with all its short positions, as there are $\frac{N}{2}$ long positions and $\frac{N}{2}$ short positions. So the maximum distance between any two portfolios is $\frac{N}{2}$. It corresponds to the diameter of the graph. Moreover, only one portfolio corresponds to the one got by permuting all the long positions with all the short positions of a given portfolio. Thus, the graph is 2-antipodal.

6.2. Computation of the spectrum of the graph generated by the 2-Weight Full Market Momentum Strategy

Let consider the graph G defined by a Full-Market Momentum strategy. Its diameter is noted $d$. From Lemma 1 (i), we know that it is a distanceregular graph. Let note its intersection table as following

$$
\left(\begin{array}{ccccc}
c_{0} & c_{1} & c_{2} & \ldots & c_{d} \\
a_{0} & a_{1} & a_{2} & \ldots & a_{d} \\
b_{0} & b_{1} & b_{2} & \ldots & b_{d}
\end{array}\right) .
$$

We know that the eigenvalues of the adjacency matrix of the distance-regular graph $\mathrm{G}$ are the eigenvalues of the following matrix $\mathrm{T}$ :

$$
T=\left(\begin{array}{cccccc}
a_{0} & b_{1} & & & & \\
c_{1} & a_{2} & b_{2} & & & \\
& c_{2} & a_{3} & \ddots & & \\
& & \ddots & \ddots & \ddots & \\
& & & & a_{d-1} & b_{d-1} \\
& & & & c_{d-1} & a_{d}
\end{array}\right) .
$$

The matrix $\mathrm{T}$ is a $(d+1) \times(d+1)$ matrix. From Lemma 1 (ii), we have $d=\frac{N}{2}$. So, for markets with a reasonable number of assets, we are able to 
compute the graph eigenvalues.

From Lemma 1 (ii), we also know that $\mathrm{G}$ is 2-antipodal. And from Dalfo et al. (2009), we know that a distance-regular graph G of diameter $d$ with spectrum

$$
\operatorname{Spec}(G)=\left(\begin{array}{cccc}
\lambda_{0} & \lambda_{1} & \ldots & \lambda_{d} \\
m_{0} & m_{1} & \ldots & m_{d}
\end{array}\right)
$$

is r-antipodal $(r \geq 2)$ if and only if its eigenvalues satisfy:

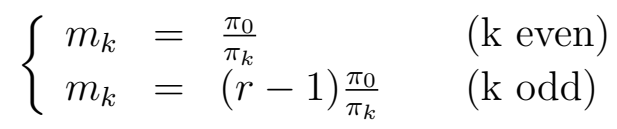

where $\pi_{k}=\prod_{i=0, i \neq k}^{d}\left|\lambda_{k}-\lambda_{i}\right|$. So we can compute the multiplicities of the eigenvalues as follows:

$$
m_{k}=\frac{\prod_{i=1}^{d}\left|\lambda_{0}-\lambda_{i}\right|}{\prod_{i=0, i \neq k}^{d}\left|\lambda_{k}-\lambda_{i}\right|}, \forall k \in\{1, \ldots, d\}
$$

\subsection{Proof of Theorem 1}

In the following, we use the bracket notation of quantum probability. Let consider a graph $G=(V, E)$ and its adjacency matrix A. Let $x, y \in V$ and $p \in \mathbb{Z}$. From proposition 2.9 in Hora and Obata (2007), we have that $<\delta_{x}\left|A^{p}\right| \delta_{y}>$ is the number of p-steps walks connecting the vertices y and $\mathrm{x}$.

So, the number of p-walks from the origin to the elements of $\Gamma_{k}$ is

$$
\sum_{x \in \Gamma_{k}}<\delta_{x}\left|A^{p}\right| \delta_{o}>
$$

Using the usual notation, we note

$$
\Phi_{k}=\frac{1}{\sqrt{\left|\Gamma_{k}\right|}} \sum_{x \in \Gamma_{k}} \delta_{x}
$$

and observing that

$$
\Phi_{0}=\delta_{o} \text { as }\left|\Gamma_{0}\right|=1
$$

the number of p-walks from the origin to the elements of $\Gamma_{k}$ is

$$
\sqrt{\left|\Gamma_{k}\right|}<\Phi_{k}\left|A^{p}\right| \Phi_{0}>
$$


Let note $\mu$ the spectral distribution of adjacency matrix A. We have

$$
\mu=\frac{1}{|\Gamma|} \sum_{i=0}^{d} m_{i} \delta_{\lambda_{i}}
$$

where $\lambda_{i}$ and $m_{i}$ are the eigenvalues, with multiplicities, of A.

As seen in section 6.1, $\mathrm{G}$ is a distance regular graph with diameter $\mathrm{d}$. In this case, from the expression (4.16) in Jafarizadeh and Salimi (2007), we have:

$$
<\Phi_{k}\left|A^{p}\right| \Phi_{o}>=\frac{1}{\left\|P_{k}\right\|} \int_{R} x^{p} P_{k}(x) \mu(d x)
$$

where $\left\{P_{k}(x)\right\}_{i=0}^{d}$ are the orthogonal polynomials defined by the three-term relation.

By integrating the last expression using the spectral distribution as defined above, we have

$$
<\Phi_{k}\left|A^{p}\right| \Phi_{o}>=\frac{1}{\left\|P_{k}\right\||\Gamma|} \sum_{i=0}^{d} m_{i} \lambda_{i}^{p} P_{k}\left(\lambda_{i}\right)
$$

So, the number of p-walks from the origin to the elements of $\Gamma_{k}$ is

$$
\frac{\sqrt{\left|\Gamma_{k}\right|}}{\left\|P_{k}\right\||\Gamma|} \sum_{i=0}^{d} m_{i} \lambda_{i}^{p} P_{k}\left(\lambda_{i}\right)
$$

Moreover, in our case, as $\mathrm{G}$ is a $d^{2}$-regular graph, the total number of p-steps walks is $d^{2 p}$. In addition, according to assumption $\mathbf{A}_{\mathbf{1}}$, the walk is random. So, each of these p-steps walks have the same probability. Consequently, the probability, for a dynamic portfolio $\gamma_{p}$ which follows a random walk on $\mathrm{G}$, to be at a distance $n$ from the original portfolio $\gamma_{o}$ after $p$ random moves, is :

$$
P\left(T O\left(\gamma_{p}, \gamma_{o}\right)=n\right)=\frac{\sqrt{\left|\Gamma_{k}\right|}}{d^{2 p}\left\|P_{k}\right\||\Gamma|} \sum_{i=0}^{d} m_{i} \lambda_{i}^{p} P_{k}\left(\lambda_{i}\right)
$$

